\title{
Usefulness of Immunohistochemistry for Microsatellite Instability Screening in Gastric Cancer
}

\author{
Yoon Sung Bae*, Hoguen Kim*, Sung Hoon $\mathrm{Noh}^{\dagger}$, and Hyunki Kim* \\ Departments of *Pathology and ${ }^{\dagger}$ Surgery, Yonsei University College of Medicine, Seoul, Korea
}

Background/Aims: The usefulness of immunohistochemistry to screen for the microsatellite instability (MSI) phenotype in gastric cancer remains unclear. Moreover, the prognostic value of MSI phenotypes in gastric cancer has been debated. Methods: The clinicopathologic parameters and survival outcomes of 203 MSI-high (MSI-H) and 261 microsatellitestable (MSS) advanced gastric cancers (AGCs) were compared. Next, we compared the immunohistochemistry results for hMLH1 and hMSH2 with those of a polymerase chain reaction (PCR)-based method. Kaplan-Meier curves and a Cox proportional hazard regression model were used to conduct survival analyses. Results: The MSI-H AGCs were correlated with older age $(p<0.001)$, female gender $(p=0.018)$, distal location $(p<0.001)$, larger size $(p=0.016)$, and intestinal type $(p<0.001)$. Multivariate analysis revealed that the MSI-H phenotype was an independent favorable factor that was related to overall survival in patients with AGC $(p<0.001)$. Compared with the PCR-based analysis, immunohistochemistry exhibited high sensitivity (91.1\%) and specificity (98.5\%) in the detection of MSI phenotypes. Conclusions: MSI-H gastric cancers have distinct clinicopathologic features and better prognoses, which suggests the necessity of MSI analysis in gastric cancer. Immunohistochemistry can be a useful and reliable screening method in the assessment of MSI status in gastric cancer. (Gut Liver 2015;9:629-635)

Key Words: Gastric cancer; Microsatellite instability; Immunohistochemistry; hMLH1; hMSH2

\section{INTRODUCTION}

The clinicopathologic characteristics of microsatellite instability-high (MSI-H) colorectal carcinomas have been thoroughly investigated and well established: younger age, proximal loca- tion, fewer lymph node metastases, and better overall survival. ${ }^{1,2}$ Several similar studies on MSI-H gastric cancer (GC) have been reported, which showed distinct clinicopathologic features including antral location, intestinal type by Lauren classification, frequent lymphoid stroma, lower prevalence of lymph node metastasis, and lower T stage, in contrast to microsatellite stable (MSS) GCs. ${ }^{3-8}$ Among the reports, a small number of studies could demonstrate favorable prognostic features of MSI-H GCs. However, a favorable effect of MSI-H phenotype was observed only in a specific subgroup in GC, which might be largely due to the limited number of study cases. ${ }^{4,5}$ If the MSI-H GCs were found to show unique clinicopathologic characteristics and perhaps even a better survival rate compared to MSS cases, it would be important for both pathologists and clinicians to analyze the MSI status of advanced gastric cancer in order to help detect patients with possible germline mutations and to estimate the clinical course.

Detection of MSI status in colorectal carcinoma is crucial and is now performed using polymerase chain reaction (PCR)-based method in many cases. Furthermore, efforts to replace the MSI test with immunohistochemistry (IHC) have been tried to overcome the shortcomings of molecular techniques, and IHC analysis with specific antibodies (hMLH1 and hMSH2) against MMR proteins has been validated as useful for investigating MSI status in colorectal cancers. ${ }^{9-12}$ Although several studies attempt to evaluate the diagnostic utility of the IHC method as a screening tool of the MSI status, ${ }^{4,6}$ the feasibility of IHC method in gastric cancer remains unclear.

To clarify the prognostic benefit of MSI-H phenotype in advanced GC (AGC) and to verify the feasibility of IHC method in predicting the MSI status of GC, we compared the clinicopathologic characteristics of a large series of MSI-H AGCs to those of MSS AGCs and evaluated the sensitivity and specificity of IHC to determine the MSI status.

Correspondence to: Hyunki Kim

Department of Pathology, Yonsei University College of Medicine, 50 Yonsei-ro, Seodaemun-gu, Seoul 120-752, Korea

Tel: +82-2-2228-1764, Fax: +82-2-362-0860, E-mail: kimhyunki@yuhs.ac

Received on March 14, 2015. Revised on June 8, 2015. Accepted on June 8, 2015.

pISSN 1976-2283 eISSN 2005-1212 http://dx.doi.org/10.5009/gnl15133

(c) This is an Open Access article distributed under the terms of the Creative Commons Attribution Non-Commercial License (http://creativecommons.org/licenses/by-nc/4.0) which permits unrestricted non-commercial use, distribution, and reproduction in any medium, provided the original work is properly cited. 


\section{MATERIALS AND METHODS}

\section{Patients and samples}

From January 2005 to December 2011, a total of 7,366 surgeries for GC patients (3,911 early GC and 3,371 AGCs) were carried out at Yonsei University Hospital. Among them, 2,959 cases (40\%) were tested for MSI phenotype by PCR-based assay on the basis of the patient's consent. Of the tested GCs, 203 cases were MSI-H type AGC. Cases that underwent neoadjuvant chemotherapy as well as recurrent cases were excluded. We additionally selected a similar number of MSS AGCs $(n=261)$. To adjust the follow-up period of MSS cases with that of MSIH AGCs, in each year, we consecutively selected the MSS cases with the same proportion of MSI-H AGCs.

Clinicopathologic parameters, including age, sex, tumor location, tumor size, gross type, histologic type, depth of invasion, lymph node metastasis, distant metastasis, lymphovascular invasion, and pTNM stage, were obtained by reviewing the medical records and pathologic reports. Tumor size was calculated by measuring the largest diameter of the tumor. Gross type was categorized by Borrmann's classification and histologic type was evaluated according to the 2010 World Health Organization (WHO) ${ }^{13}$ and Lauren's classifications. ${ }^{14}$ Due to similarity with intestinal type on survival analysis, cases with mixed type on Lauren's classification were regarded as intestinal type (data not shown). Pathologic TNM stage was evaluated in accordance with the seventh 2010 American Joint Committee on Cancer/International Union Against Cancer (AJCC/UICC) staging system. ${ }^{15}$ The clinical outcomes of patients were observed from the date of operation to the date of death or to May 1, 2013. Informed consent for MSI analysis was obtained from all patients. Authorization for the use of included tissues for research purposes was obtained from the Institutional Review Board of Yonsei University College of Medicine (approval number: 4-2013-0460).

\section{Microsatellite analysis}

Genomic DNA from tumor and nonneoplastic areas was extracted using the QIAamp DNA mini kit (Qiagen, Hilden, Germany) and tested with five microsatellite markers, two mononucleotide repeats (BAT25 and BAT26), and three dinucleotide repeats (D2S123, D5S346, and D17S250), as recommended by the National Cancer Institute. PCR was performed using a fluorescence-labeled multiprimer, HotStarTaq polymerase (Qiagen), and the GeneAmp PCR system 2700 (Applied Biosystems, Foster City, CA, USA), for which the process was composed of initial denaturation ( 15 minutes at $95^{\circ} \mathrm{C}$ ), followed by 30 cycles of denaturation ( 1 minute at $\left.94^{\circ} \mathrm{C}\right)$, annealing $\left(1\right.$ minute at $57^{\circ} \mathrm{C}$ ), and extension ( 1 minute at $72^{\circ} \mathrm{C}$ ). Amplification was performed with a final 5 minutes at $72^{\circ} \mathrm{C}$ and the amplified PCR products were analyzed using the automated ABI PRISM sequencer model 3100 genetic analyzer (Applied Biosystems). MSI was determined by size variation and the presence of additional bands in the PCR products from tumor DNA. Tumors were designated as MSI-H when at least two out of the five markers showed instability, and as MSS when none of them showed instability. ${ }^{16}$ MSI-low cases, which showed instability in only one marker, were excluded from this study.
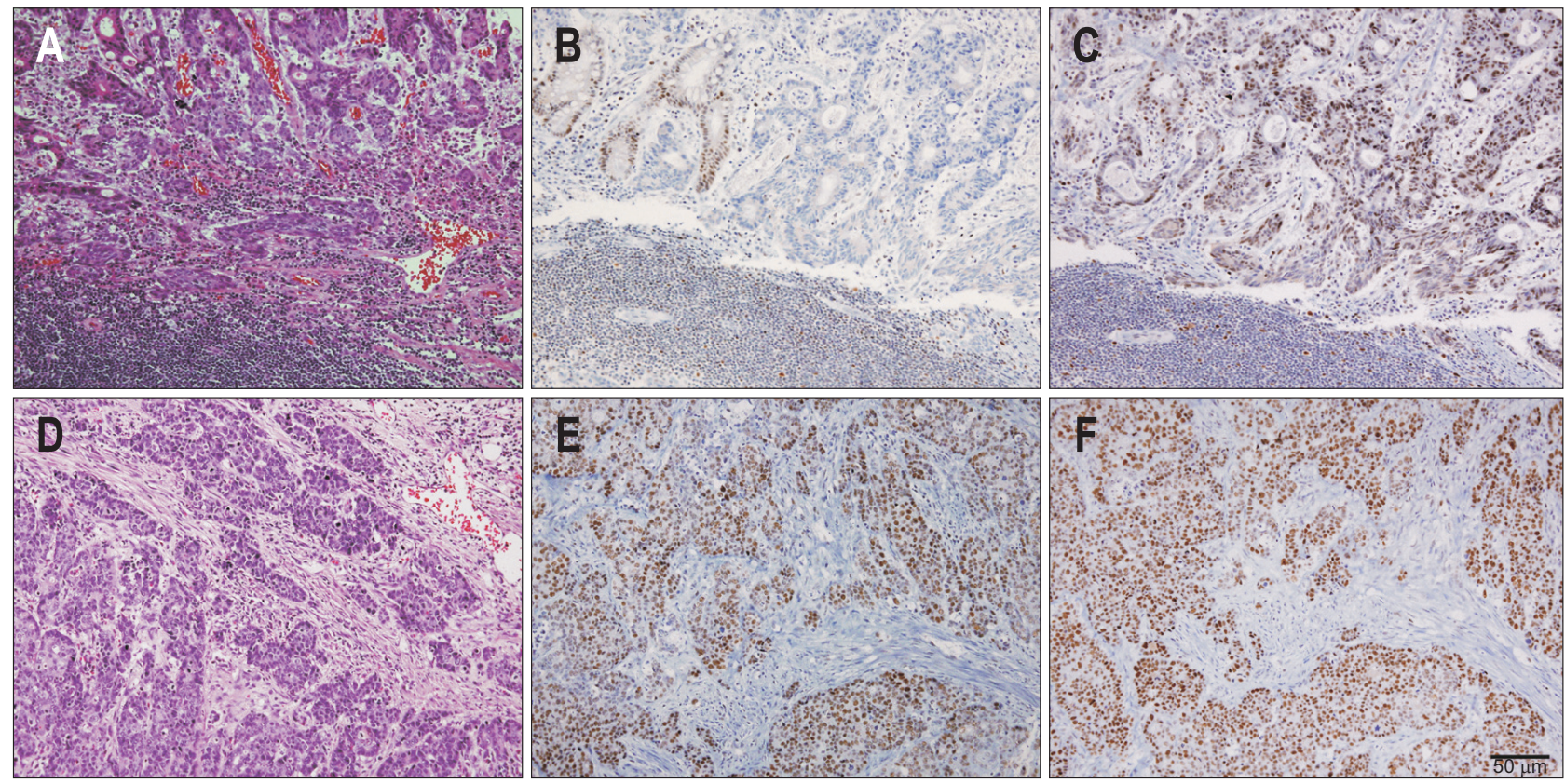

Fig. 1. Representative histology and immunohistochemistry results. A microsatellite instability-high (MSI-H) case (A) exhibiting the loss of the expression of hMLH1 (B) and the intact expression of hMSH2 (C). Stromal lymphocytes were used as an internal positive control. The intact expression of hMLH1 (E) and hMSH2 (F) was evident in a microsatellite stable case (D). 


\section{Tissue microarray construction}

To select representative tumor areas, hematoxylin and eosin (H\&E)-stained slides were reviewed by a pathologist before constructing the tissue arrays. Tissue cores of $3 \mathrm{~mm}$ in diameter were transplanted from individual donor blocks to tissue array blocks. Each tissue array block contained 29 cases and one normal gastric mucosa as a landmark and internal control, with a total of 464 cases in 16 array blocks. H\&E and cytokeratin IHC were performed to confirm the presence of tumor.

\section{Immunohistochemistry}

Paraffin-embedded tissue blocks were cut into $4 \mu \mathrm{m}$ sections. IHC was performed using a Ventana XT automated stainer (Ventana Corp., Tucson, AZ, USA) with antibody to hMLH1 (readyto-use, clone M1; Roche, Indianapolis, IN, USA) and hMSH2 (1:300, clone G219-1129; BD Pharmingen, San Jose, CA, USA). Sections were deparaffinized using EZ Prep solution (Ventana Corp.). CC1 standard (pH 8.4 buffer containing Tris/borate/ EDTA) was used for antigen retrieval and blocked with inhibitor D $\left(3 \% \mathrm{H}_{2} \mathrm{O}_{2}\right)$ for 4 minutes at $37^{\circ} \mathrm{C}$. Slides were incubated with primary antibody for 40 minutes at $37^{\circ} \mathrm{C}$ followed by a universal secondary antibody for 20 minutes at $37^{\circ} \mathrm{C}$. Slides were incubated in streptavidin-horseradish peroxidase (SA-HRP) D for 16 minutes at $37^{\circ} \mathrm{C}$ and then the substrate, 3,3'-diaminobenzidine tetrahydrochloride (DAB) $\mathrm{H}_{2} \mathrm{O}_{2}$, was added for 8 minutes followed by hematoxylin and bluing reagent counterstaining at $37^{\circ} \mathrm{C}$. A loss of MMR protein expression was designated when none of the neoplastic epithelial cells showed nuclear staining, while normal expression was defined as the presence of nuclear staining of tumor cells, irrespective of the proportion or intensity (Fig. 1). Infiltrating lymphocytes, stromal cells and adjacent nonneoplastic epithelium served as internal positive controls. Two pathologists (Yoon Sung Bae and Hyunki Kim) assessed the IHC results without awareness of the MSI status of each case.

\section{Statistical analysis}

The sensitivity and specificity of IHC detection were calculated using molecular MSI results as standards. Sensitivity and specificity were defined as loss of hMLH1 and/or hMSH2 expression in MSI-H and their intact expression in MSS GCs, respectively. The chi-square test and t-test were performed to determine the possible correlation between MSI phenotype and clinicopathologic parameters. Survival curves were analyzed using the Kaplan-Meier method, followed by the log-rank test for estimating significant differences. Multivariate survival analysis was performed using the Cox proportional hazard regression model. All of the data were analyzed using IBM SPSS version 20.0 (IBM Corp., Armonk, NY, USA), and the results were considered to be statistically significant when the $p$-values were less than 0.05 .

\section{RESULTS}

\section{Clinicopathologic characteristics according to MSI phe- notype}

The patients were composed of 315 males and 149 females,

Table 1. Clinicopathologic Characteristics according to the Microsatellite-Stable and Microsatellite Instability-High Phenotype in Gastric Cancer

\begin{tabular}{|c|c|c|c|c|}
\hline Variable & Total & $\begin{array}{l}\text { MSS GC } \\
(\mathrm{n}=261)\end{array}$ & $\begin{array}{c}\text { MSI-H GC } \\
(\mathrm{n}=203)\end{array}$ & $\mathrm{p}$-value \\
\hline Age, yr & & & & $<0.001$ \\
\hline$\leq 60$ & 209 & $140(67.0)$ & $69(33.0)$ & \\
\hline$>60$ & 255 & $121(47.5)$ & $134(52.5)$ & \\
\hline Gender & & & & 0.018 \\
\hline Male & 315 & $189(60.0)$ & $126(40.0)$ & \\
\hline Female & 149 & $72(48.3)$ & 77 (51.7) & \\
\hline Tumor size, $\mathrm{cm}$ & & & & $<0.001$ \\
\hline$\leq 6$ & 258 & $158(61.2)$ & $100(38.8)$ & \\
\hline$>6$ & 206 & $103(50.0)$ & $103(50.0)$ & \\
\hline Tumor location & & & & $<0.001$ \\
\hline Upper and middle & 169 & $126(74.6)$ & $43(25.4)$ & \\
\hline Lower & 295 & $135(45.8)$ & $160(54.2)$ & \\
\hline Borrmann's type & & & & $<0.001$ \\
\hline I and II & 163 & 66 (40.5) & 97 (59.5) & \\
\hline III and IV & 301 & $195(64.8)$ & $106(35.2)$ & \\
\hline Histologic type & & & & 0.001 \\
\hline Well and moderately & 189 & $89(47.1)$ & $100(52.9)$ & \\
\hline Poorly & 275 & $172(62.5)$ & $103(37.5)$ & \\
\hline Lauren's classification & & & & $<0.001$ \\
\hline Intestinal & 290 & $133(45.9)$ & $157(54.1)$ & \\
\hline Diffuse & 174 & $128(73.6)$ & $46(26.4)$ & \\
\hline Lymphovascular invasion & & & & 0.972 \\
\hline Absent & 203 & $114(56.2)$ & 89 (43.8) & \\
\hline Present & 261 & $147(56.3)$ & $114(43.7)$ & \\
\hline Depth of invasion & & & & $<0.001$ \\
\hline pT2 and pT3 & 272 & $129(47.4)$ & $143(52.6)$ & \\
\hline pT4 & 192 & $132(68.8)$ & $60(31.3)$ & \\
\hline Lymph node metastasis & & & & 0.030 \\
\hline Absent & 149 & $73(49.0)$ & $76(51.0)$ & \\
\hline Present & 315 & $188(59.7)$ & $127(40.3)$ & \\
\hline Distant metastasis & & & & 0.028 \\
\hline Absent & 435 & 239 (54.9) & $196(45.1)$ & \\
\hline Present & 29 & 22 (75.9) & $7(24.1)$ & \\
\hline pTNM stage & & & & $<0.001$ \\
\hline I and II & 218 & $97(44.5)$ & $121(55.5)$ & \\
\hline III and IV & 246 & $164(66.7)$ & $82(33.3)$ & \\
\hline
\end{tabular}

Data are presented as number (\%).

MSS, microsatellite-stable; GC, gastric cancer; MSI-H, microsatellite instability-high. 
with a mean age of 61 years (range, 26 to 88 years). The mean tumor size was $5.9 \mathrm{~cm}$ (range, 0.7 to $25 \mathrm{~cm}$ ). In accordance with WHO classification, 189 (40.7\%) were cases of well to moderately differentiated tubular adenocarcinoma, 227 (48.9\%) were cases of poorly differentiated tubular adenocarcinoma and signet ring cell carcinoma, and 48 (10.3\%) were defined as other. The 48 other cases were composed of mucinous carcinoma, carcinoma with lymphoid stroma (medullary carcinoma), and adenosquamous carcinoma. Those cases were regarded as poorly differentiated tumor by definition. Of the 464 cases, 272 (58.6\%) and 192 (41.4\%) were pT2/3 and pT4, respectively. Lymph node metastasis was observed in 315 cases (67.9\%) and 29 cases (6.3\%) had distant metastasis. Grouped by pTNM stage, 58 cases (12.5\%) were stage IB, 160 (34.5\%) were stage II, 217 (46.8\%) were stage III, and 29 (6.3\%) were stage IV. The mean follow-up period was 51 months (range, 1 to 100 months).

The correlation between clinicopathologic parameters and MSI phenotype is summarized in Table 1 . The mean age was 58.4 years in the MSS group and 64.2 years in the MSI-H group. The mean tumor size was $5.7 \mathrm{~cm}$ in the former group and 6.2 $\mathrm{cm}$ in the latter. GCs with MSI-H phenotype were associated with older age $(\mathrm{p}<0.001)$, female gender $(\mathrm{p}=0.018)$, distal tumor location $(p<0.001)$, larger tumor size $(p<0.001)$, Type I or II gross type according to Borrmann's classification $(\mathrm{p}<0.001)$, and well to moderately differentiated $(\mathrm{p}=0.001)$ and intestinal histologic types $(\mathrm{p}<0.001)$ according to WHO and Lauren's classification. MSI-H GCs were also characterized by a lower pT stage $(\mathrm{p}<0.001)$ and lower nodal metastasis $(\mathrm{p}=0.030)$.

\section{Survival analysis}

The results of univariate and multivariate survival analyses are summarized in Table 2. Upon univariate analysis, MSS phenotype $(p<0.001)$, larger tumor size $(>6 \mathrm{~cm}, \mathrm{p}<0.001)$, Borrmann's type III/IV ( $\mathrm{p}<0.001)$, poorly differentiated histology $(p=0.011)$, diffuse type by Lauren's classification $(p<0.001)$, deeper depth of invasion (pT4, p<0.001), presence of lymph node metastasis $(p<0.001)$, presence of metastasis $(p<0.001)$, and presence of lymphovascular invasion $(\mathrm{p}<0.001)$ were correlated with poor survival outcomes. The multivariate analyses using those significant variables showed that the MSS phenotype was

Table 2. Univariate and Multivariate Survival Analysis

\begin{tabular}{|c|c|c|c|c|c|c|}
\hline \multirow{2}{*}{ Factor } & \multicolumn{3}{|c|}{ Univariate analysis } & \multicolumn{3}{|c|}{ Multivariate analysis } \\
\hline & HR & $95 \% \mathrm{CI}$ & p-value & $\mathrm{HR}$ & $95 \% \mathrm{CI}$ & $\mathrm{p}$-value \\
\hline \multicolumn{7}{|l|}{ MSI } \\
\hline MSI-H vs MSS & 2.785 & $1.767-4.391$ & $<0.001$ & 1.798 & $1.095-2.935$ & 0.020 \\
\hline \multicolumn{7}{|l|}{ Age, yr } \\
\hline$\leq 60$ vs $>60$ & 0.928 & $0.634-1.358$ & 0.700 & - & - & - \\
\hline \multicolumn{7}{|l|}{ Gender } \\
\hline Male vs female & 1.277 & $0.861-1.896$ & 0.224 & - & - & - \\
\hline \multicolumn{7}{|l|}{ Tumor size, cm } \\
\hline$\leq 6$ vs $>6$ & 2.435 & $1.646-3.601$ & $<0.001$ & 1.823 & $1.202-2.764$ & 0.005 \\
\hline \multicolumn{7}{|l|}{ Tumor location } \\
\hline U/M vs L & 0.913 & $0.616-1.352$ & 0.650 & - & - & - \\
\hline \multicolumn{7}{|l|}{ Borrmann's type } \\
\hline I/II vs III/IV & 3.109 & $1.871-5.167$ & $<0.001$ & 1.745 & $1.022-2.981$ & 0.041 \\
\hline \multicolumn{7}{|l|}{ Histologic type } \\
\hline Well/moderately vs poorly & 1.711 & $1.130-2.591$ & 0.011 & 0.664 & $0.349-1.264$ & 0.213 \\
\hline \multicolumn{7}{|l|}{ Lauren's classification } \\
\hline Intestinal vs diffuse & 2.491 & $1.696-3.659$ & $<0.001$ & 1.696 & $0.935-3.076$ & 0.082 \\
\hline \multicolumn{7}{|l|}{ Depth of invasion } \\
\hline pT2/3 vs pT4 & 4.076 & $2.691-6.173$ & $<0.001$ & 2.107 & $1.329-3.341$ & 0.002 \\
\hline \multicolumn{7}{|l|}{ Lymph node metastasis } \\
\hline Absent vs present & 7.155 & $3.478-14.718$ & $<0.001$ & 3.707 & $1.758-7.816$ & 0.001 \\
\hline \multicolumn{7}{|l|}{ Distant metastasis } \\
\hline Absent vs present & 4.199 & $2.553-6.906$ & $<0.001$ & 2.462 & $1.471-4.121$ & 0.001 \\
\hline \multicolumn{7}{|l|}{ Lymphovascular invasion } \\
\hline Absent vs present & 3.136 & $1.976-4.978$ & $<0.001$ & 1.999 & $1.241-3.219$ & 0.004 \\
\hline
\end{tabular}

HR, hazard ratio; CI, confidence interval; MSI-H, microsatellite instability-high; MSS, microsatellite stable; U/M, upper or middle; L, lower. 
an independent risk factor for worse survival outcomes (hazard ratio, 1.798; 95\% confidence interval, 1.095 to 2.935; $\mathrm{p}=0.020$ ). In terms of other parameters, tumor size ( $p=0.005)$, Borrmann's type $(p=0.041)$, lymphovascular invasion $(p=0.004)$, depth of invasion $(p=0.002)$, lymph node metastasis $(p=0.001)$, and distant metastasis ( $\mathrm{p}=0.001$ ) were correlated with patient survival. The survival benefit in MSI-H GCs was also demonstrated by Kaplan-Meier survival curves (Fig. 2A). Even when grouped according to stage, these tumors showed a better prognosis, particularly stage II ( $\mathrm{p}=0.003$ ) (Fig. 2B). Stage III tumors, although not statistically significant, were found to have a tendency towards better overall survival ( $\mathrm{p}=0.116$ ) (Fig. 2C). Statistical significance was achieved when the intestinal type cases were selectively included ( $\mathrm{p}=0.010$ ) (Fig. 2D). Furthermore, the survival benefit of MSI-H GCs was additionally analyzed by separating cases into an hMLH1 loss group and an hMSH2 loss group. Loss of hMLH1 expression was significantly associated with a better prognosis $(\mathrm{p}<0.001$ on univariate analysis and $\mathrm{p}=0.03$ on multivariate analysis), while loss of hMSH2 expression tended to show survival benefit without statistical power (Supplementary Table 1, Fig. 3).

\section{Immunohistochemical results}

As outlined in Table 3, of the 203 MSI-H cases, 185 cases showed loss of expression of either hMLH1 or hMSH2; 179 for hMLH1, 15 for hMSH2, and nine for both of them, indicating a sensitivity of 91.1\%. In 261 MSS cases, 257 cases normally reacted to both proteins, for a specificity of $98.5 \%$. Of the total 464 cases, 22 cases showed a discrepancy between IHC and PCR results, including four cases of MSS and 18 cases of MSI-H, determined on the basis of PCR-based analysis.
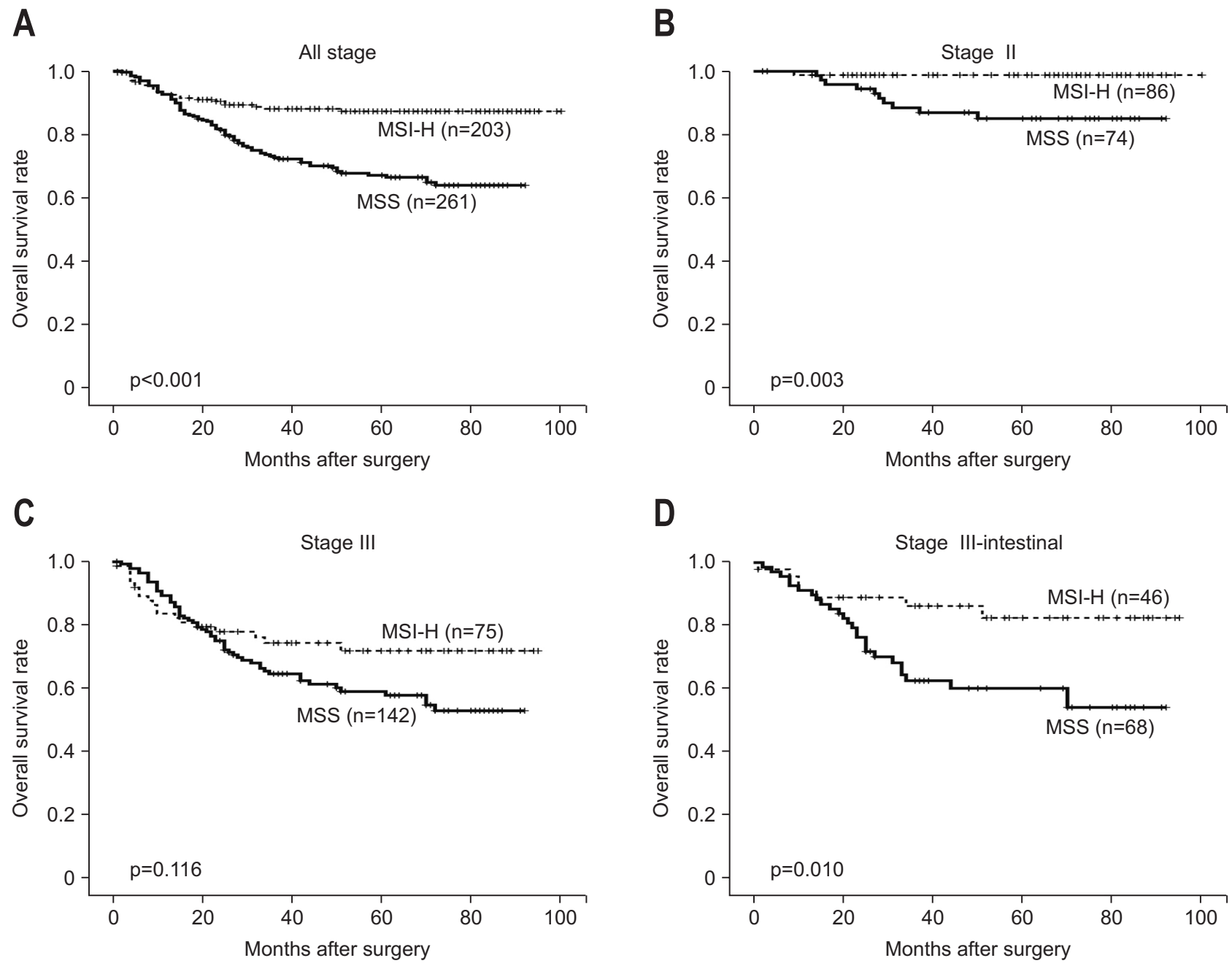

Fig. 2. Kaplan-Meier analysis of the overall survival outcomes of advanced gastric cancer (AGC) patients according to polymerase chain reaction (PCR)-based microsatellite instability (MSI) analyses. (A) MSI-high (MSI-H) AGCs were associated with significantly better survival rates than the microsatellite stable (MSS) AGCs ( $\mathrm{p}<0.001$ ), which was also observed in stage II ( $\mathrm{p}=0.003)$ (B). (C) In stage III, the MSI-H AGCs exhibited a trend toward good prognoses compared with the MSS AGCs, although there was no statistical significance $(\mathrm{p}=0.116)$. (D) The intestinal-type MSI-H AGCs exhibited better overall survival than did the intestinal-type MSS AGCs, including in stage III ( $\mathrm{p}=0.010)$. 
A

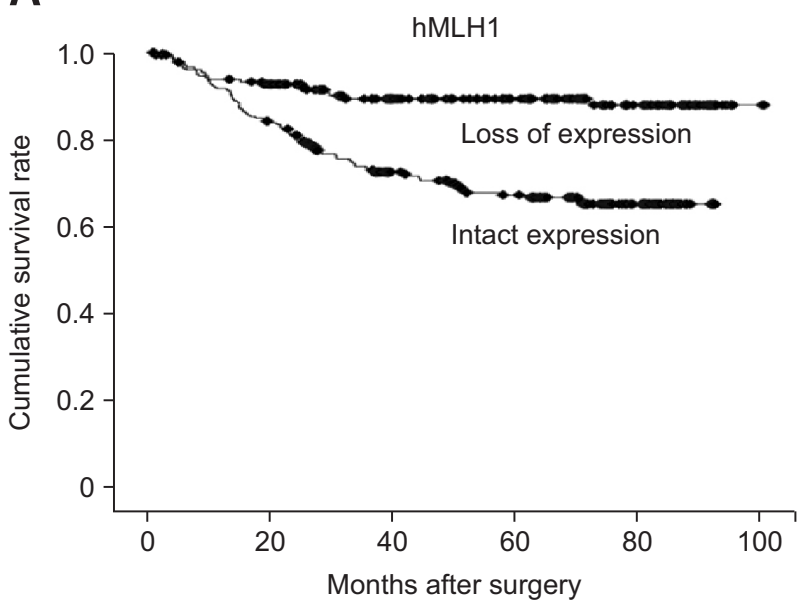

B

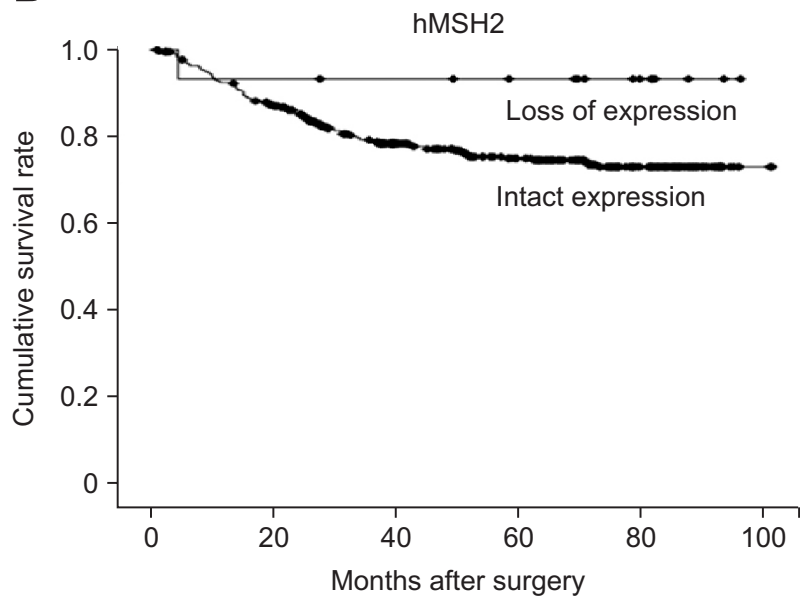

Fig. 3. Kaplan-Meier survival curves according to hMLH1 and hMSH2 expressions. The loss of hMLH1 expression was significantly associated with a survival benefit (A), whereas the loss of hMSH2 expression was associated with a tendency toward a better prognosis that did not reach statistical significance (B).

Table 3. Immunohistochemical Results for hMLH1 and hMSH2 in the Screening of Microsatellite Instability Status

\begin{tabular}{|c|c|c|c|c|c|c|}
\hline \multirow{2}{*}{ Group } & \multicolumn{2}{|c|}{ hMLH1 } & \multicolumn{2}{|c|}{ hMSH2 } & \multirow{2}{*}{$\begin{array}{l}\text { hMLH1-loss or } \\
\text { hMSH2-loss }\end{array}$} & \multirow{2}{*}{$\begin{array}{l}\text { hMLH1-intact and } \\
\text { hMSH2-intact }\end{array}$} \\
\hline & Intact & Loss & Intact & Loss & & \\
\hline MSI-H (n=203) & 24 (11.8) & 179 (88.2) & 188 (92.6) & $15(7.4)$ & $185\left(91.1^{*}\right)$ & $18(8.9)$ \\
\hline MSS (n=261) & 257 (98.5) & $4(1.5)$ & 203 (100.0) & 0 & $4(1.5)$ & 257 (98.5*) \\
\hline
\end{tabular}

Data are presented as number (\%).

MSI-H, microsatellite instability-high; MSS, microsatellite stable.

*The values of 91.1\% and 98.5\% were the sensitivity and specificity, respectively, of the immunohistochemistry for the screening of the MSI phenotype of gastric cancers.

\section{DISCUSSION}

The previous studies showed that GCs with the MSI-H phenotype have relatively distinct clinicopathologic characteristics, including older age, distal location, larger tumor size, intestinal type according to Lauren's classification, gross type II according to Borrmann's classification, a lower rate of lymph node metastasis, and lower pTNM stage. ${ }^{3-7,17}$ Similar results were found in this study. However, the survival benefits of MSI-H phenotype remains a matter of debate, because the numbers of patients with MSI-H GCs were not high enough to evaluate. ${ }^{4,5}$ With a large scale series of 203 MSI-H AGCs, we could demonstrate the survival benefit of MSI-H GCs.

Using the Cox proportional hazard regression analysis and Kaplan-Meier survival curves, we demonstrated that MSI-H GCs had a better overall survival outcome than MSS GCs. As summarized in Table 1, however, the $\mathrm{pT}$ and $\mathrm{pN}$ stages were different with statistical significance in both groups, which required stratified analysis. Therefore, we reanalyzed after stratifying for pTNM stage. In stage II, MSI phenotype was still a crucial factor in predicting increased survival rates. Also in stage III, although not statistically significant, GCs with MSI-H had a tendency towards favorable outcomes. Interestingly, if the cases are confined to intestinal type according to Lauren's classification, MSI-H GCs showed a better survival rate than MSS GCs with statistical significance even in stage III. Similar results were reported in our previous study. ${ }^{5}$ In that study, MSI-H GCs had more favorable outcomes than MSS GCs of the intestinal type, but not the diffuse type. The results of stage IV were hard to interpret because of the limited number of cases. ${ }^{5}$

MSI status could be a significant factor not only in colorectal cancers but also in GCs, given that MSI-H phenotype is a specific entity in GCs and has survival benefits. Although PCRbased molecular examination has been regarded as a standard diagnostic method in the identification of microsatellite instability in many tumors, including colorectal carcinoma, ${ }^{16}$ IHC is more simple, rapid, and universally available than the molecular testing. The usefulness of IHC in predicting MSI status has previously been validated extensively in colorectal cancers and was found to have high sensitivity and specificity. ${ }^{10-12,18}$

In terms of GCs, although there have been several attempts to verify the efficacy of IHC, the results were not satisfactory, especially in terms of sensitivity. ${ }^{3,4,6}$ In our study, the results of IHC staining for antibodies against hMLH1 and hMSH2 showed a 
satisfactory reliability (91.1\% sensitivity and 98.5\% specificity). The discrepancy between the data from previous studies and ours might have resulted partly from the larger number of cases studied. Additionally, as we could control the environment during IHC procedures with automated IHC stainer, we could obtain high-quality, more reproducible, and more standardized IHC results. A small subset of MSI-H cancers may show mutation of other MMR genes rather than MLH1 and MSH2, such as PMS2 and MSH6, which could be a reason of slight low sensitivity in this study. ${ }^{19}$ Even though the status of PMS2 and MSH6 also could be detected using IHC, it would not yield a cost-effective result.

In conclusion, MSI-H GCs have distinct clinicopathologic features and a better prognosis, which suggests the necessity of MSI analysis in GCs. From a routine diagnostic standpoint, IHC may be a useful and reliable screening method in predicting MSI status in GCs and will reduce the number of cases that need to be tested by PCR-based analysis.

\section{CONFLICTS OF INTEREST}

No potential conflict of interest relevant to this article was reported.

\section{ACKNOWLEDGEMENTS}

This research was supported by the Basic Science Research Program through the National Research Foundation of Korea (NRF) funded by the Ministry of Education, Science and Technology (2012R1A1A1004403) and by a faculty research grant for Yonsei University College of Medicine for 2012 (6-20120044).

\section{REFERENCES}

1. Kim H, Jen J, Vogelstein B, Hamilton SR. Clinical and pathological characteristics of sporadic colorectal carcinomas with DNA replication errors in microsatellite sequences. Am J Pathol 1994;145: 148-156

2. Gryfe R, Kim H, Hsieh ET, et al. Tumor microsatellite instability and clinical outcome in young patients with colorectal cancer. $\mathrm{N}$ Engl J Med 2000;342:69-77.

3. Lee HS, Choi SI, Lee HK, et al. Distinct clinical features and outcomes of gastric cancers with microsatellite instability. Mod Pathol 2002;15:632-640.

4. Beghelli S, de Manzoni G, Barbi S, et al. Microsatellite instability in gastric cancer is associated with better prognosis in only stage II cancers. Surgery 2006;139:347-356.

5. Kim H, An JY, Noh SH, Shin SK, Lee YC, Kim H. High microsatellite instability predicts good prognosis in intestinal-type gastric cancers. J Gastroenterol Hepatol 2011;26:585-592.
6. Seo HM, Chang YS, Joo SH, et al. Clinicopathologic characteristics and outcomes of gastric cancers with the MSI-H phenotype. J Surg Oncol 2009;99:143-147.

7. Falchetti M, Saieva C, Lupi R, et al. Gastric cancer with high-level microsatellite instability: target gene mutations, clinicopathologic features, and long-term survival. Hum Pathol 2008;39:925-932.

8. Fang WL, Chang SC, Lan YT, et al. Microsatellite instability is associated with a better prognosis for gastric cancer patients after curative surgery. World J Surg 2012;36:2131-2138.

9. Chaves P, Cruz C, Lage P, et al. Immunohistochemical detection of mismatch repair gene proteins as a useful tool for the identification of colorectal carcinoma with the mutator phenotype. J Pathol 2000;191:355-360.

10. Lindor NM, Burgart LJ, Leontovich 0, et al. Immunohistochemistry versus microsatellite instability testing in phenotyping colorectal tumors. J Clin Oncol 2002;20:1043-1048.

11. Jourdan F, Sebbagh N, Comperat E, et al. Tissue microarray technology: validation in colorectal carcinoma and analysis of p53, hMLH1, and hMSH2 immunohistochemical expression. Virchows Arch 2003;443:115-121.

12. Hendriks Y, Franken P, Dierssen JW, et al. Conventional and tissue microarray immunohistochemical expression analysis of mismatch repair in hereditary colorectal tumors. Am J Pathol 2003;162:469477.

13. Bosman FT; World Health Organization; International Agency for Research on Cancer. WHO classification of tumours of the digestive system. 4th ed. Lyon: International Agency for Research on Cancer, 2010

14. Lauren P. The two histological main types of gastric carcinoma: diffuse and so-called intestinal-type carcinoma. An attemp at a histo-clinical classification. Acta Pathol Microbiol Scand 1965;64:31-49.

15. Edge SB; American Joint Committee on Cancer; American Cancer Society; American College of Surgeons. AJCC cancer staging manual. 7th ed. New York: Springer, 2010.

16. Umar A, Boland CR, Terdiman JP, et al. Revised Bethesda Guidelines for hereditary nonpolyposis colorectal cancer (Lynch syndrome) and microsatellite instability. J Natl Cancer Inst 2004;96: 261-268.

17. Kim SH, Ahn BK, Nam YS, Pyo JY, Oh YH, Lee KH. Microsatellite instability is associated with the clinicopathologic features of gastric cancer in sporadic gastric cancer patients. J Gastric Cancer 2010;10:149-154.

18. Shia J, Ellis NA, Klimstra DS. The utility of immunohistochemical detection of DNA mismatch repair gene proteins. Virchows Arch 2004;445:431-441

19. de Jong AE, van Puijenbroek M, Hendriks Y, et al. Microsatellite instability, immunohistochemistry, and additional PMS2 staining in suspected hereditary nonpolyposis colorectal cancer. Clin Cancer Res 2004;10:972-980. 